\title{
ESTUDO NEUROANATOMICO DO RAMO ORBITOFRONTAL DA ARTERIA CEREBRAL ANTERIOR EM CEREBROS HUMANOS
}

\author{
VICENTE PAULO JORGE LEMOS * \\ MARTA MARIA DAS CHAGAS MEDEIROS *" \\ REGINA COELI MARQUES DE CARVALHO **
}

Tem recebido várias denominações a artéria classicamente considerada como o primeiro ramo cortical do segmento pós-comunicante da artéria cerebral anterior: orbitária ${ }^{3}$, pré-frontal ${ }^{2}$, frontal inferior ${ }^{5,11}$, orbitofrontal 16, frontobasal ${ }^{4}$. Apesar da variada terminologia, esse ramo da artéria cerebral anterior tem sido apenas brevemente descrito. Ring e Waddington ${ }^{8}$, na dissecação de 50 hemisférios cerebrais, observou que a artéria orbitofrontal aparecia individualizada em $72 \%$ dos casos, era dupla em $12 \%$, múltipla em $4 \%$ e estava ausente em 12\%; nestes últimos, em dois hemisférios o território era suprido pela artéria homônima contralateral e nos 4 hemisférios restantes a área era suprida por ramo aberrante da artéria cerebral média. Marino ?, em 40 hemisférios cerebrais, registrou frequência de $100 \%$; segundo ele, a artéria orbitofrontal supre a porção orbital do primeiro giro frontal e o seu território compreende, de modo aproximado, às áreas 10,11 e 25 de Brodmann. Perlmutter e Rhoton 8 , no estudo de 50 hemisférios cerebrais, encontrou a artéria orbitofrontal em $82 \%$ dos casos como ramo individualizado da artéria cerebral anterior; nos restantes $18 \%$, ela originava-se em tronco comum com a artéria frontopolar. A pormenorizada caracterização morfológica deste e de outros pequenos ramos das artérias cerebrais, num passado não muito remoto, era árida e irrelevante. Hoje, reveste-se de certa importância como fonte de dados ao progressivo desenvolvimento e aprimoramento das técnicas microneurocirúrgicas e no que concerne às interpretações de aspectos filogenéticos do cérebro e a correspondente evolução do seu padrão vascular.

No presente estudo da artéria orbitofrontal, pretende-se: determinar a frequência deste vaso em ambos os hemisférios cerebrais, visto que os dados existentes na literatura são predominantemente oriundos de observações em hemisférios cerebrais isolados, sem referências especificas quanto ao lado; identificar possiveis variações da origem e do trajeto do segmento extracerebral da artéria e, também, sua condição de primeiro ramo cortical do segmento pós-comunicante da artéria cerebral anterior, considerando que isto nem sempre ocorre, especialmente nos casos da origem pós-comunicante da artéria recorrente de Heubner ${ }^{8}$; analisar comparativamente os achados desta observação e os já registrados na literatura, visando à interpretação do significado filogenético da

Trabalho do Departamento de Morfologia do Centro de Ciencias da Saúde da Universidade Federal do Cearé (UFCe): * Professor Adjunto; ** Bolsista do Plano de Iniclação a Pesquisa da UFCe. 
artéria orbitofrontal. Dentre as muitas denominações, a de artéria orbitofrontal foi a adotada neste trabalho pela conotação descritiva que ela encerra, principalmente quanto à indicação do território suprido.

\section{MATERIAL E METODOS}

Foram estudados 50 encéfalos humanos procedentes de cadáveres de pessoas cujas idades variavam entre 16 e 76 anos e entre as quais era acentuado o predomínio do sexo masculino.

O material foi fixado injetando-se solução de formal a $10 \%$ nos troncos das três artérias cerebrais, no terceiro ventriculo e nos ventrículos laterais. Em 20 encéfalos, previamente à fixação pelo formol, a artéria cerebral anterior foi injetada com solução de vinilite (resina VYHH), corada em vermelho, para facilitar a identificação e a dissecção dos ramos proximais da referida artéria. As dissecções da artéria orbitofrontal foram realizadas sob lupa estereoscópica Jena, com ampliação de 6,3X.

\section{RESULTADOS}

A artéria orbitofrontal, na qualidade ramo direito da artéria cerebral anterior, estava presente em $90 \%(45 / 50)$ dos casos no hemisfério direito e em 92\% (46/50) no hemisfério esquerdo. Com maior frequêencia, em ambos os lados, a artéria surgiu como ramo simples do segmento pós-comunicante da artéria cerebral anterior; em alguns casos houve duplicidade unilateral e, em outros, a artéria era inexistente. Quanto a origem, esta invariavelmente ocorreu na porção inicial ascendente do segmento pós-comunicante da artéria cerebral anterior, ora entre a origem da artéria frontopolar e o ramo comunicante anterior do círculo arterial do cérebro, ora entre as origens da artéria frontopolar e da artéria recorrente de Heubner, nos casos em que esta última também apareceu como ramo pós-comunicante da artéria cerebral anterior. Os aspectos quantitativos destas observações estão indicados na tabela 1.

Com relação ao percurso do segmento extracerebral da artéria orbitofrontal não foram percebidas diferenças significativas quanto ao sexo e, também, nos dois hemisférios cerebrais de um mesmo indíviduo. Quanto ao trajeto, partindo da origem, a artéria dirigiu-se inferiormente e, num curto trajeto recorrente, alcançou a porção caudal do sulco olfatório, no qual penetrou medialmente ao tracto olfatório. Na profundidade desse sulco, descrevia alça ascendente e no sentido rostral, após o que emergia lateralmente ao tracto olfatório, ramificando-se na porção medial da superfície orbitária do lobo frontal.

No âmbito das limitações de uma disseç̧ão simples sob lupa estereoscópica, observou-se que a artéria orbitofrontal emitia inúmeros pequenos ramos corticais ao longo do seu curso na superfície da regiáo orbitária do lobo frontal e na profundidade do sulco olfatório. Desses ramos, o mais constante ocorria logo que a artéria penetrava no sulco olfatório; com muita frequência ele era paralelo ao tracto olfatório, para o qual destinava finissimos ramos colaterais. De modo bastante aproximado, a extensão do território vascular da artéria orbitofrontal abrangia as áreas 11 e 25 
de Brodmann, em coincidência aos achado de Marino 7 à exceção da área 10 que, em todos os exemplares observados neste trabalho, se constituiu território da artéria frontopolar. Nos casos de ausência da artéria orbitofrontal, seu território era suprido em um caso por ramos da artéria recorrente de Heubner e, em outro, por ramo aberrante da artéria cerebral média; nos demais casos, a irrigação era dada por ramos da artéria frontopolar.

\begin{tabular}{lccccc}
\hline $\begin{array}{c}\text { Hemisfério } \\
\text { cerebral }\end{array}$ & $\begin{array}{c}\text { Artéria } \\
\text { simples }\end{array}$ & $\begin{array}{c}\text { Artéria } \\
\text { dupla }\end{array}$ & Ausência & $\begin{array}{c}\text { Ponto de origem entre: } \\
\text { CoA/Fp }\end{array}$ & $\begin{array}{c}\text { RecH/Fp } \\
\text { RecH }\end{array}$ \\
\hline Direito & $66 \%(33 / 50)$ & $24 \%(12 / 50)$ & $10 \%(5 / 50)$ & $52 \%(26 / 50)$ & $38 \%(19 / 50)$ \\
Esquerdo & $60 \%(30 / 50)$ & $32 \%(16 / 50)$ & $8 \%(4 / 50)$ & $68 \%(34 / 50)$ & $24 \%(12 / 50)$
\end{tabular}

Tabela 1 - I'requência e lacalizaçáo do ponto de origem da artéria orbitofrontal no segmento pbs-comunicalnte da artérla cerebral anterior, em 50 encéfalos humanos adultos, de ambos os sexos. Legenda: CoA - comunidante anterior; I'p - frontopolar; RecH - recorrente de Heubner.

\section{COMENTARIOS}

A frequência e a constância do território da artéria orbitofrontal, registradas neste trabalho, contribuem para caracterizá-la como entidade anatômica bem definida. Quanto ao significado filogenético, existem dados na literatura que constituem fundamentação teórica à proposição de hipótese de homologia entre a artéria orbitofrontal humana e os ramos orbitário anterior e orbitário posterior, ambos colaterais da artéria cerebral anterior, descritos por Watts em primatas sub-humanos (orangotango, chimpanzé e gorila) ${ }^{12}$.

Segundo Abbie 1 , as artérias cerebral anterior e cerebral média mantêm conexũes anastomóticas ao nivel do primórdio do neopálio nos répteis. Nos mamiferos sub-primatas - pelo "princípio da constância funcional" - esses canais teriam permanecido durante a expansão dorso-posterior do neopálio entre o hipocampo e o lobo piriforme, em torno da fissura endorrinal. Consequentemente, o desenvolvimento neopalial teria determinado deslocamento progressivo do território da artéria cerebral desde seu campo anterior primitivo, que incluia a porção lateral do bulbo olfatório. Neste processo evolutivo - pelo "princípio da conveniência de fonte" - a artéria cerebral anterior teria usurpado esse território da artéria cerebral média, pelo maior desenvolvimento dos primitivos canais anastomóticos e assumindo desse modo a suplência de ambos os lados do bulbo olfatório e da porção medial da superfície orbitária do lobo frontal. De acordo com o relatado e que é também baseado em Abbie ${ }^{1}$, é atraente a idéia de que os ramos orbitário anterior e orbitário posterior dos primatas sub-humanos teriam evoluído a partir daqueles canais anastomóticos entre as artérias cerebral anterior e cerebral média, com nítido predomínio de fluxo sanguíneo da artéria cerebral anterior. 
Considerando a correspondência dos territórios de vascularização da artéria orbitofrontal humana e dos ramos orbitário anterior e orbitário posterior dos primatas sub-humanos, ambos representados pelas porções mediais da superfície orbitária do lobo frontal, incluindo tracto e bulbo olfatórios, caberiam as seguintes interpretações dos resultados alcançados: a ocorrência da artéria orbitofrontal simples no cérebro humano seria explicada segundo o "princípio da economia de distribuição" (quando duas ou mais artérias terminais suprem uma mesma área, há tendência para formação de um tronco arterial único) ${ }^{1}$; os casos de artéria orbitofrontal dupla representariam resquício de padrão filogenético primitivo, estabelecido durante o desenvolvimento embrionário e mantido no adulto; a ausência da artéria orbitofrontal e o suprimento do seu território feito por um ou mais ramos aberrantes da artéria cerebral média indicaria, também, vestígio do antigo território desta artéria.

\section{RESUMO}

Estudo neuroanatômico da artéria orbitofrontal, considerando frequência, origem e distribuição, foi realizado em 50 encéfalos humanos, dissecados sob lupa estereoscópica. Os resultados alcançados contribuem para caracterizar este ramo da artéria cerebral anterior como entidade anatômica bem definida. Do ponto de vista filogenético, foi considerada a hipótese de homologia entre a artéria orbitofrontal e os ramos orbitário posterior e orbitário anterior dos primatas sub-humanos.

SUMMARY

Neuroanatomic study of the orbitofrontal branch of the anterior cerebral artery in human brains.

The neuroanatomic study of the orbitofrontal branch of the anterior cerebral artery was performed by simple and careful dissection in 50 human brains. The observation was carried out with stereoscopic magnifying glass. The results with regard to frequency and origin of the artery were the following: the orbitofrontal artery was present in $90 \%(45 / 50)$ of the cases on the right hemisphere and $92 \%(46 / 50)$ on the left hemisphere; in 26 cases $(52 \%)$ on the right side and 34 cases $(68 \%)$ on the opposite side, the artery arose between anterior communicating and frontopolar arteries; in 19 cases (38\%) on the right side and 12 cases $(24 \%)$ on the left side the vessel was not the classical first branch of the postcommunical segment of the anterior cerebral artery, it arose between the Heubner artery and the frontopolar artery. With regard at filogenetic signification of the orbitofrontal artery it has been formulated the hypothesis that the artery is homologous to posterior and anterior orbital branches of the anterior cerebral artery described by Watts in sub-human primates. 
1. ABBIE, A. A. - The morphology of the fore-brain arteries with especial reference to the evolution of the basal ganglia. J. Anat. (London) 68:433, 1934.

2. CRITCHLEY, M. - The anterior cerebral artery and its syndromes. Brain 53: 120, 1930.

3. FOIX, C. \& HILLEMAND, P. - Les syndromes de l'artère cérébrale anterieure. L'Encéphale 20:209, 1925.

4. KRAYENBUHL, H. \& YASARGIL, M. G. - Radiological anatomy and topography of the cerebral arteries In: VINKEN, P. J. \& BRUYN, G. W. - Handbook Clinical Neurology 11:79, 1972. North-Holland, Amsterdam.

5. LAZORTHES, G.; GAUBERT, J. \& POULHES, J. - La distribution centrale et corticale de l'artère cérébrale antérieure. tude anatomique et incidences neurochirurgicales. Neurochir. (Paris) 2:237, 1956.

6. LEMOS, V.P. - Ramo recorrente da artéria cerebral anterior: um estudo neuroanatômico do segmento extracerebral. Rev. bras. Pesq. méd. biol. 10:21, 1977.

7. MARINO Jr., R. - The anterior cerebral artery: I. Anatomo-radiological study of its cortical territories. Surg. Neurol. 5:81, 1976.

8. PERLMUTTER, D. \& RHOTON Jr., A. L. - Microsurgical anatomy of the distal anterior cerebral artery. J. Neurosurg. 49:204, 1978.

9. RING, B. A. \& WADDINGTON, M. M. - Roentgenographic anatomy of the pericallosal arteries. Am. J. Roentgenol. 104:109, 1968.

10. SALAMON, G. \& HUANG, Y. P. - Radiologic anatomy of the brain. Springer, Berlin, 1976.

11. SHELLSHEAR, J. A. - A contribution to our knowledge of the arterial supply of the cerebral cortex in man. Brain 50:236, 1927.

12. WATTS, J. W. - A comparative study of the anterior cerebral artery and the circle of Willis. J. Anat. (London) 68:534, 1934.

Departamento de Morfologia - Centro de Ciencias da Saude - Universidade Federal do Ceard - Cairoa Postal 688 - Fortaleza 60.000 - $C E$ - Brasil. 